\title{
Some Relations and Values For the Generalized Riemann Zeta Function
}

\author{
By Eldon R. Hansen and Merrell L. Patrick
}

1. Introduction. In this paper we derive several relations among values of the generalized Riemann Zeta function $\zeta(s, a)$. We show that there is a "fundamental domain" for the variables $s$ and $a$ so that values outside this domain can be obtained from those inside by algebraic operations, using values of the Gamma and trigonometric functions.

We give a table of values of the Zeta function which were calculated using these relations to supplement the formulas commonly used for such calculations. Table 1 gives $\zeta(s, a)$ to seventeen decimal places for $a=\frac{1}{4}$ and $\frac{3}{4}$ and for $s=$ $-\frac{61}{3}\left(\frac{1}{3}\right) \frac{64}{3}$. The ordinary Riemann Zeta function is also given to seventeen decimal places for $s=0\left(\frac{1}{3}\right) \frac{64}{3}$ in Table 2. These tables are useful in diffraction theory $[10],[11]$.

2. Relations among Zeta Functions. Consider the defining relation

$$
\zeta(s, a)=\sum_{n=0}^{\infty} \frac{1}{(n+a)^{s}}
$$

which holds for $\operatorname{Re}(s)>1$ and all values of $a$ except zero or negative integers. (One usually assumes $s$ complex but $a$ real.) We first note that

$$
\begin{aligned}
\zeta(s, a+1) & =\sum_{n=0}^{\infty} \frac{1}{(n+a+1)^{8}} \\
& =\sum_{n=0}^{\infty} \frac{1}{(n+a)^{s}}-\frac{1}{a^{8}} \\
& =\zeta(s, a)-a^{-s} .
\end{aligned}
$$

By analytic continuation, this relation holds for all $s$. Thus if $\zeta(s, a)$ is known for all $s$, but only for values of $a$ satisfying $a_{0}<a \leqq a_{0}+1$ for some $a_{0}$, then we can calculate $\zeta(s, a)$ for any value of $a$ by algebraic operations. One usually specifies $a_{0}=0$, so that the "fundamental interval" is $0<a \leqq 1$.

We now show that this fundamental interval in $a$ need only be of length $\frac{1}{2}$. In the defining relation (1), let $a=(1 / q)-b$ where $q$ is an integer. Then

$$
\begin{aligned}
\zeta\left(s, \frac{1}{q}-b\right) & =q^{s} \sum_{n=0}^{\infty} \frac{1}{(n q+1-b q)^{8}} \\
& =q^{s}\left[\sum_{n=0}^{\infty} \frac{1}{(n+1-b q)^{s}}-\sum_{r=2}^{q} \sum_{n=0}^{\infty} \frac{1}{(n q+r-b q)^{s}}\right] \\
& =q^{s} \zeta(s, 1-b q)-\sum_{r=2}^{q} \zeta\left(s, \frac{r}{q}-b\right) .
\end{aligned}
$$

Received January 10, 1962. 
Therefore

$$
\sum_{r=1}^{q} \zeta\left(s, \frac{r}{q}-b\right)=q^{8} \zeta(s, 1-b q) .
$$

By analytic continuation, this result holds for all $s$. This relation was obtained by Powell [7] for the special case $s=\frac{1}{2}$.

Equation (3) yields many interesting and useful results. For example, let $b=\frac{1}{2}-c$. Then, for $q=2$, we obtain

$$
\zeta(s, c)=2^{s} \zeta(s, 2 c)-\zeta\left(s, c+\frac{1}{2}\right) .
$$

For $c=\frac{1}{2}$ this yields the well known result

$$
\zeta\left(s, \frac{1}{2}\right)=\left(2^{s}-1\right) \zeta(s),
$$

where $\zeta(s)$ is the ordinary Riemann Zeta function defined by $\zeta(s, 1)=\zeta(s)$.

Now assume that $\zeta(s, a)$ is known for all $s$ and for all $a$ in the interval $\frac{1}{2}<a \leqq 1$. Then equation (4) shows that, if $a_{1}$ is such that $0<a_{1} \leqq \frac{1}{2}$, then $\zeta\left(s, a_{1}\right)$ can be expressed in terms of values assumed known. We merely apply relation (4) repeatedly as needed. An inductive proof can be easily obtained but will not be given here. As an example of the use of equation (4), consider the case $a_{1}=\frac{1}{8}$. From equation (4),

$$
\zeta\left(s, \frac{1}{8}\right)=2^{8} \zeta\left(s, \frac{1}{4}\right)-\zeta\left(s, \frac{5}{8}\right)
$$

The argument $\frac{1}{4}$ is not in the desired interval, so we use (4) to obtain

$$
\zeta\left(s, \frac{1}{4}\right)=2^{8} \zeta\left(s, \frac{1}{2}\right)-\zeta\left(s, \frac{3}{4}\right)
$$

Again, we do not know $\zeta\left(s, \frac{1}{2}\right)$, so we use (4) to obtain

$$
\zeta\left(s, \frac{1}{2}\right)=2^{s} \zeta(s, 1)-\zeta(s, 1)=\left(2^{s}-1\right) \zeta(s) .
$$

Combining (6), (7) and (8), we have

$$
\zeta\left(s, \frac{1}{8}\right)=2^{28}\left(2^{s}-1\right) \zeta(s)-2^{8} \zeta\left(s, \frac{3}{4}\right)-\zeta\left(s, \frac{5}{8}\right) .
$$

All the functions in the right member are assumed known, hence $\zeta\left(s, \frac{1}{8}\right)$ is obtained by algebraic operations.

For special values of $a$, the more general relation (3) may be used more fruitfully than the special form (4).

We now see that $\frac{1}{2}<a \leqq 1$ is a fundamental interval for $\zeta(s, a)$. We next show that for rational values of $a$, we can obtain $\zeta(s, a)$ for $\operatorname{Re}(s)<\frac{1}{2}$ from values for $\operatorname{Re}(s) \geqq \frac{1}{2}$.

Consider the Hurwitz formula (see [1], page 269)

$$
\frac{(2 \pi)^{s}}{2 \Gamma(s)} \zeta(1-s, a)=\cos \frac{\pi s}{2} \sum_{n=1}^{\infty} \frac{\cos 2 \pi n a}{n^{s}}+\sin \frac{\pi s}{2} \sum_{n=1}^{\infty} \frac{\sin 2 \pi n a}{n^{s}},
$$

which is valid for $\operatorname{Re}(s)>1$ and $0<a \leqq 1$. Let $a=p / q$, where $p$ and $q$ are in- 
tegers (which need not be relatively prime). Then

$$
\begin{aligned}
\frac{(2 \pi)^{s}}{2 \Gamma(s)} \zeta(1 & \left.-s, \frac{p}{q}\right)=\cos \frac{\pi s}{2} \sum_{n=1}^{\infty} \frac{\cos 2 \pi n p / q}{n^{s}}+\sin \frac{\pi s}{2} \sum_{n=1}^{\infty} \frac{\sin 2 \pi n p / q}{n^{s}} \\
& =\cos \frac{\pi s}{2} \sum_{r=0}^{q-1} \sum_{n=1}^{\infty} \frac{\cos 2 \pi(q n-r) p / q}{(q n-r)^{s}}+\sin \frac{\pi s}{2} \sum_{r=0}^{q-1} \sum_{n=1}^{\infty} \frac{\sin 2 \pi(q n-r) p / q}{(q n-r)^{s}} \\
& =\frac{1}{q^{s}} \cos \frac{\pi s}{2} \sum_{r=0}^{q-1} \sum_{n=1}^{\infty} \frac{\cos 2 \pi r p / q}{(n-r / q)^{s}}-\frac{1}{q^{s}} \sin \frac{\pi s}{2} \sum_{r=0}^{q-1} \sum_{n=1}^{\infty} \frac{\sin 2 \pi r p / q}{(n-r / q)^{s}} \\
& =\frac{1}{q^{8}} \sum_{r=0}^{q-1}\left[\cos \frac{\pi s}{2} \cos \frac{2 \pi r p}{q}-\sin \frac{\pi s}{2} \sin \frac{2 \pi r p}{q}\right] \sum_{n=0}^{\infty} \frac{1}{(n+1-r / q)^{s}} .
\end{aligned}
$$

Replacing $r$ by $q-r$ and simplifying,

$$
\zeta(1-s, p / q)=\frac{2 \Gamma(s)}{(2 \pi q)^{8}} \sum_{r=1}^{q} \cos \pi\left(\frac{s}{2}-\frac{2 r p}{q}\right) \zeta(s, r / q) .
$$

By analytic continuation, this result holds for all $s$. Thus, if $\zeta(s, a)$ is known for $\operatorname{Re}(s) \geqq \frac{1}{2}$ and all rational $a$, we can compute $\zeta(s, a)$ for all $s$ and all rational $a$, $0<a \leqq 1$, from equation (10). Conversely, if we know $\zeta(s, a)$ for all rational $a, 0<a \leqq 1$, and for $\operatorname{Re}(s) \leqq \frac{1}{2}$, we can compute $\zeta(s, a)$ from equation (10) for; all $s$ except for integer values. The right hand side of equation (10) contains $\Gamma(s)$, which is infinite for $s$ a negative integer. Hence equation (10) cannot, in general, be used directly to yield $\zeta(s, a)$ for $s$ a positive integer. However equations (2), (4), and (10) enable us to find $\zeta(s, a)$ for all $s$ and all rational $a$ from values of $\zeta(s, a)$ for $\operatorname{Re}(s) \geqq \frac{1}{2}$ and $\frac{1}{2}<a \leqq 1$.

Let us now consider some special cases of equation (10). For $p=1$ and $q=2$, we again obtain equation (5). For $p=q=1$, we obtain the Riemann relation

$$
\zeta(1-s)=\frac{2 \Gamma(s)}{(2 \pi)^{s}} \cos \frac{\pi s}{2} \zeta(s)
$$

For the general case $p=q$, we obtain, by using relation (11),

$$
\sum_{r=1}^{q-1} \zeta(s, r / q)=\left(q^{s}-1\right) \zeta(s)
$$

which also follows from equation (3). If we also write equation (12) with $s$ replaced by $1-s$, then the resulting equation can be combined with equations (11) and (12) to yield

$$
\pi^{-s / 2} \Gamma(s / 2)\left(q^{s}-1\right) \sum_{r=1}^{q-1} \zeta(s, r / q)
$$

$$
=\pi^{-(1-s) / 2} \Gamma\left(\frac{1-s}{2}\right)\left(q^{1-s}-1\right) \sum_{r=1}^{\prime-1} \zeta(1-s, r / q) .
$$

That is, the members of this equation are invariant under replacement of $s$ by $1-s$. Hence we may regard equation (13) as a generalization of the Riemimn 
relation (11), which is often written as

$$
\Gamma\left(\frac{1-s}{2}\right) \pi^{-(1-s) / 2} \zeta(1-s)=\Gamma\left(\frac{s}{2}\right) \pi^{-s / 2} \zeta(s) .
$$

Next substitute $q=4$ and $p=1$ and 3 in equation (10). In this case, we obtain, using equations (5) and (11)

$$
\zeta\left(1-s, \frac{1}{2} \pm \frac{1}{4}\right)=\frac{2-2^{s}}{4^{s}} \zeta(1-s) \pm \frac{2 \Gamma(s)}{(8 \pi)^{8}} \sin \frac{\pi s}{2}\left[\zeta\left(s, \frac{3}{4}\right)-\zeta\left(s, \frac{1}{4}\right)\right]
$$

Subtracting this equation, using the lower sign from the equation using the upper sign, we obtain

$$
\zeta\left(1-s, \frac{3}{4}\right)-\zeta\left(1-s, \frac{1}{4}\right)=\frac{4 \Gamma(s)}{(8 \pi)^{s}} \sin \frac{\pi s}{2}\left[\zeta\left(s, \frac{3}{4}\right)-\zeta\left(s, \frac{1}{4}\right)\right] .
$$

Now the Dirichlet $L$-function is defined to be

$$
L(s)=\sum_{n=0}^{\infty} \frac{(-1)^{n}}{(2 n+1)^{s}}
$$

for $\operatorname{Re}(s)>1$. This can be expressed as

$$
L(s)=4^{-8}\left[\zeta\left(s, \frac{1}{4}\right)-\zeta\left(s, \frac{3}{4}\right)\right] .
$$

Hence equation (15) can be rewritten as

$$
L(1-s)=(2 / \pi)^{s} \Gamma(s) \sin \frac{\pi s}{2} L(s) .
$$

This relation is derived (but misprinted) by Titchmarsh (see [6], page 66).

For $m$ equal to a positive integer,

$$
\zeta(-m, a)=-\frac{B_{m+1}(a)}{(m+1)},
$$

where $B_{m}(a)$ is the $m$ th Bernoulli polynomial in $a$. (See [1], page 267.) Hence, letting $s \rightarrow-2 m$ in equation (14), and using the fact that $B_{m}(1-a)$ $=(-1)^{m} B_{m}(a)$, we obtain

$$
\zeta\left(2 m+1, \frac{1}{2} \pm \frac{1}{4}\right)=2^{2 m}\left(2^{2 m+1}-1\right) \zeta(2 m+1) \pm \frac{2^{6 m+1} \pi^{2 m+1}}{(2 m+1) !} B_{2 m+1}\left(\frac{1}{4}\right)
$$

From these equations, we find that

$$
L(2 m+1)=-\frac{(2 \pi)^{2 m+1}}{2(2 m+1) !} B_{2 m+1}\left(\frac{1}{4}\right) .
$$

Many other interesting results can be obtained by looking at special cases of equation (10).

The method used to derive equations (3) and (10) can also be applied to the more general function

$$
\zeta(s, a, z)=\sum_{n=0}^{\infty} \frac{z^{n}}{(n+a)^{s}}
$$

discussed by Mitchell [8], Lerch [9] and others. 
3. Calculation of the Table. In the discussion above we showed, by using equation (10), that we could find $\zeta(s, a)$ for the $\operatorname{Re}(s) \leqq \frac{1}{2}$ and rational $a, 0<a \leqq 1$, provided we know $\zeta(s, a)$ for $\operatorname{Re}(s)>\frac{1}{2}$ and rational $a, 0<a \leqq 1$. The equation

$$
\begin{aligned}
\zeta(s, a)=\sum_{n=0}^{N-1} \frac{1}{(n+a)^{s}}-\frac{(N+a)^{1-s}}{1-s}+\frac{(N+a)^{-s}}{2} \\
\quad-\sum_{n=1}^{\infty} \frac{B_{n}}{(2 n) !} \frac{\Gamma(1-s)}{\Gamma(2-s-2 n)}(N+a)^{-s-(2 n-1)},
\end{aligned}
$$

where $B_{1}=\frac{1}{6}, B_{2}=-\frac{1}{30}, B_{3}=\frac{1}{42}, B_{4}=-\frac{1}{80}, B_{5}=\frac{5}{86}, \cdots$ are Bernoulli numbers, is a representation of $\zeta(s, a)$ for $\operatorname{Re}(s)>0, s \neq 1$, and $0<a \leqq 1$. We use this asymptotic series to calculate $\zeta\left(s, \frac{1}{4}\right), \zeta\left(s, \frac{3}{4}\right)$, and $\zeta(s, 1)=\zeta(s)$ for $s=\frac{2}{3}\left(\frac{1}{3}\right) \frac{64}{3}$. It is not necessary to use this expression to calculate $\zeta\left(s, \frac{1}{2}\right)$ because of equation (8). With these values of $\zeta\left(s, \frac{1}{4}\right), \zeta\left(s, \frac{3}{4}\right)$, and $\zeta(s, 1)$ for $s=\frac{2}{3}\left(\frac{1}{3}\right) \frac{64}{3}$, we calculate $\zeta\left(s, \frac{1}{4}\right)$ and $\zeta\left(s, \frac{3}{4}\right)$, using $(14)$, for $s=-\frac{61}{3}\left(\frac{1}{3}\right) \frac{1}{3}$ with the exception $s=0$. For $s=0$ we use the identity $\zeta(0, a)=\frac{1}{2}-a$. The Gamma function, $\Gamma(s)$, appearing on the right side of equation (10) is calculated by making use of the asymptotic series (see [1] Section 12.33)

$$
\log \Gamma(s)=\left(s-\frac{1}{2}\right) \log s-s+\frac{1}{2} \log (2 \pi)+\sum_{r=1}^{\infty} \frac{(-1)^{r-1} B_{r}}{2 r(2 r-1) s^{2 r-1}}
$$

where $B_{r}$ are the Bernoulli numbers. This expression holds for $|\arg s| \leqq \frac{1}{2} \pi-\Delta$ and $0<\Delta<\frac{1}{4} \pi$. Using this relation in conjunction with the equation $\Gamma(s+1)=$ $s \Gamma(s)$, we obtain the desired values of the Gamma function.

Equation (17), for $a=1$, is equivalent to the expression used by Gram [2] and by Haselgrove [3] in preparing their tables of the ordinary Riemann Zeta function.

4. Methods of Checking the Table Values. Equation (16) was used to check the table entries for negative integer values of $s$, while Hurwitz's formula (9) was used to check the entries for $s=-\frac{61}{3}\left(\frac{1}{3}\right)-\frac{28}{3}$. For positive integer values of $s$, the values of $\zeta(s)$ agree exactly with the table in [5], page xxv. Our table was not checked for other values of $s$, since no other simple method seemed to present itself. Note, however, the table for negative values of $s$ was calculated using the entries for positive values of $s$. Hence, it seems safe to assume that the table entries for positive values of $s$ are correct to at least as many digits as the table entries for corresponding negative values of $s$.

5. Comments Regarding the Calculation of the Table. The table shown below was calculated using a precision of seventy binary digits, which is approximately equivalent to twenty-one decimal digits.

It was impossible to enter most of the values of $s$ exactly in the computer, since $1 / 3$ does not have a finite binary representation. Therefore, we should consider the errors introduced in our table from using a truncated binary representation. We were able to show, by using a truncated Taylor series, that the errors so introduced did not affect the accuracy of the table. The various calculations involved in showing the insignificance of these errors were not included in this paper, since they were quite straightforward. 
6. The Table of Values. The tabular data have been listed to seventeen significant decimal digits. The decimal point is located immediately to the left of the left-most digit of each entry, while the two-digit integer at the right denotes the exponent of the power of ten by which the entry is to be multiplied.

Lockheed Missiles and Space Company

Palo Alto, California

1. E. T. Whittaker \& G. N. Watson, A Course in Modern Analysis, fourth edition, Cambridge, 1952 .

2. J. P. Gram, "Tafeln für die Riemannsche Zetafunktion," Kungl. Danske Vid. Selsk. skr. (8), v. 10,1925, p. 313-325. 1960 .

3. C. B. HASElgrove, Tables of the Riemann Zeta Function, Cambridge University Press,

4. R. Hensman, Tables of the Generalized Riemann Zeta Function, Report No. T 2111, Telecommunications Research Establishment, Ministry of Supply, Great Malvern, Worcestershire, 1948 .

5. British Association for the Advancement of Science, Mathematical Tables, Vol. I, ('ircular and Hyperbolic Functions, third edition, Cambridge University Press, 1951.

6. E. C. TITCHMarsh, Introduction to the Theory of Fourier Integrals, second edition, Oxford University Press, 1948.

7. E. O. POWELL, "A table of the generalized Riemann zeta function in a particular case," Quart. J. Mech. Appl. Math. v. 5, 1952, p. 116-119.

8. K. MitchelL, "Tables of a function and related functions," Phil. Mag., v. 40, 1949, p. $351-368$.

9. E. IERCH, "Note sur la fonction $R(w, x, s)=\sum_{0}^{\infty} \frac{\mathrm{e}^{2 k \pi i x}}{(w+k)^{\mathrm{s}}}, "$ Acta. Math. (Stockholm), v. 11, 1887, p. 19-24.

10. H. Bremmer, Terrestrial Radio Waves, Elsevier Publishing Co., New York, 1949.

11. Nelson Logan, General Research in Diffraction Theory, v. I., Lockheed Missiles and Sipace Division Report *288087, December 1959. 
TABLE 1

\begin{tabular}{|c|c|c|c|c|}
\hline$s$ & \multicolumn{2}{|l|}{$\zeta\left(s, \frac{1}{4}\right)$} & \multicolumn{2}{|l|}{$\zeta\left(s, \frac{3}{4}\right)$} \\
\hline $64 / 3$ & $\begin{array}{llll}69814 & 63658 & 33156 & 77\end{array}$ & 13 & $\begin{array}{lll}46276 & 4164209957 \quad 54\end{array}$ & 03 \\
\hline 21 & $\begin{array}{llll}43980 & 46511 & 10400 & 92\end{array}$ & 13 & $\begin{array}{llll}42044 & 91481 & 42985 & 79\end{array}$ & 03 \\
\hline $62 / 3$ & $\begin{array}{llll}27705 & 95688 & 87832 & 77\end{array}$ & 13 & 38200340499972472 & 03 \\
\hline $61 / 3$ & 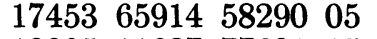 & 13 & $\begin{array}{llll}34707 & 31296 & 92456 & 16\end{array}$ & 03 \\
\hline 20 & $\begin{array}{lllll}10995 & 11627 & 77601 & 15\end{array}$ & 13 & $\begin{array}{llll}31533 & 68689 & 82531 & 57\end{array}$ & 03 \\
\hline $59 / 3$ & $\begin{array}{llll}69264 & 89222 & 19591 \quad 87\end{array}$ & 12 & $\begin{array}{llll}28650 & 25632 & 40314 & 70\end{array}$ & 03 \\
\hline $58 / 3$ & $43634 \quad 14786 \quad 4573582$ & 12 & $\begin{array}{llll}26030 & 48587 & 06422 & 96\end{array}$ & 03 \\
\hline 19 & $27487 \quad 7906944014 \quad 41$ & 12 & $23650 \quad 26655 \quad 19854 \quad 06$ & 03 \\
\hline $56 / 3$ & $\begin{array}{llll}17316 & 22305 & 54910 & 39\end{array}$ & 12 & $\begin{array}{llll}21487 & 69390 & 40321 & 69\end{array}$ & 03 \\
\hline $55 / 3$ & $\begin{array}{llll}10908 & 53696 & 61447 & 33\end{array}$ & 12 & $\begin{array}{llll}19522 & 86640 & 47054 & 77\end{array}$ & 03 \\
\hline 18 & $\begin{array}{llll}68719 & 47673 & 60180 & 15\end{array}$ & 11 & $17737 \quad 70232 \quad 63351 \quad 39$ & 03 \\
\hline $53 / 3$ & $\begin{array}{llll}43290 & 55763 & 87431 & 22\end{array}$ & 11 & $\begin{array}{llll}1611577333 & 52502 \quad 14\end{array}$ & 03 \\
\hline $52 / 3$ & $2727134241 \quad 53785 \quad 57$ & 11 & $\begin{array}{llll}14642 & 15330 & 71921 & 73\end{array}$ & 03 \\
\hline 17 & $\begin{array}{llll}17179 & 86918 & 40225 & 19\end{array}$ & 11 & 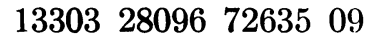 & 03 \\
\hline $50 / 3$ & $\begin{array}{llll}10822 & 63940 & 97051 & 87\end{array}$ & 11 & 12086835090354536 & 03 \\
\hline $49 / 3$ & $\begin{array}{llll}68178 & 35603 & 86554 & 48\end{array}$ & 10 & $\begin{array}{lllll}10981 & 62111 & 35873 & 48\end{array}$ & 03 \\
\hline 16 & $\begin{array}{llll}42949 & 67296 & 02814 & 98\end{array}$ & 10 & $\begin{array}{lllll}99774 & 68117 & 34460 & 20\end{array}$ & 02 \\
\hline $47 / 3$ & $2705659852 \quad 4505563$ & 10 & $\begin{array}{llll}90651 & 35226 & 87845 & 72\end{array}$ & 02 \\
\hline $46 / 3$ & 17044589009925196 & 10 & 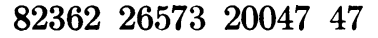 & 02 \\
\hline 15 & $\begin{array}{llll}10737 & 41824 & 03518 & 96\end{array}$ & 10 & $\begin{array}{lllll}74831 & 14030 & 73469 & 44\end{array}$ & 02 \\
\hline $44 / 3$ & $\begin{array}{lllll}67641 & 49631 & 42966 & 14\end{array}$ & 09 & $67988 \quad 67020 \quad 7991481$ & 02 \\
\hline $43 / 3$ & $\begin{array}{lllll}42611 & 47252 & 80800 & 17\end{array}$ & 09 & $\begin{array}{llll}61771 & 88735 & 02511 & 35\end{array}$ & 02 \\
\hline 14 & 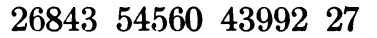 & 09 & $\begin{array}{llll}56123 & 58191 & 92793 & 72\end{array}$ & 02 \\
\hline $41 / 3$ & $\begin{array}{llll}16910 & 37408 & 23656 & 51\end{array}$ & 09 & $\begin{array}{llll}50991 & 77593 & 55328 & 03\end{array}$ & 02 \\
\hline $40 / 3$ & $1065286813 \quad 6104594$ & 09 & 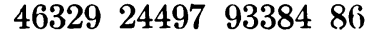 & 02 \\
\hline 13 & $\begin{array}{llll}67108 & 86405 & 50022 & 12\end{array}$ & 08 & $\begin{array}{lllll}42093 & 08367 & 45511 & 12\end{array}$ & 02 \\
\hline $38 / 3$ & $\begin{array}{llll}42275 & 93525 & 33217 & 14\end{array}$ & 08 & 38244310935548072 & 02 \\
\hline $37 / 3$ & $\begin{array}{lllll}26632 & 17039 & 13370 & 57\end{array}$ & 08 & $\begin{array}{llll}34747 & 51134 & 84446 & 15\end{array}$ & 02 \\
\hline 12 & 16777216068779631 & 08 & $\begin{array}{llll}31570 & 50939 & 08321 & 59\end{array}$ & 02 \\
\hline $35 / 3$ & $\begin{array}{llll}10568 & 98387 & 26213 & 03\end{array}$ & 08 & $\begin{array}{lllll}28684 & 07349 & 76745 & 21\end{array}$ & 02 \\
\hline $34 / 3$ & $\begin{array}{lllll}66580 & 42661 & 71997 & 90\end{array}$ & 07 & $26061 \quad 64725 \quad 71060 \quad 80$ & 02 \\
\hline 11 & $\begin{array}{llll}4194304086 & 03548 & 00\end{array}$ & 07 & $\begin{array}{llll}23679 & 10527 & 14066 & 46\end{array}$ & 02 \\
\hline $32 / 3$ & $\begin{array}{llll}26422 & 46042 & 34022 & 87\end{array}$ & 07 & $2151453144 \quad 7837237$ & 02 \\
\hline $31 / 3$ & $\begin{array}{llll}16645 & 10745 & 38096 & 69\end{array}$ & 07 & $\begin{array}{llll}19548 & 01769 & 36192 & 33\end{array}$ & 02 \\
\hline 10 & $\begin{array}{lllll}10485 & 76107 & 68311 & 48\end{array}$ & 07 & $\begin{array}{llll}17761 & 48118 & 13370 & 04\end{array}$ & 02 \\
\hline $29 / 3$ & $\begin{array}{lllll}66056 & 16034 & 78926 & 59\end{array}$ & 06 & $\begin{array}{llll}16138 & 49852 & 76111 & 40\end{array}$ & 02 \\
\hline $28 / 3$ & $\begin{array}{llll}41612 & 77864 & 98520 & 10\end{array}$ & 06 & $\begin{array}{llll}14664 & 15539 & 12355 & 32\end{array}$ & 02 \\
\hline 9 & $26214 \quad 413492172408$ & 06 & 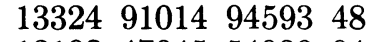 & 02 \\
\hline $26 / 3$ & 16514051736108533 & 06 & $1210847045 \quad 5408094$ & 02 \\
\hline $25 / 3$ & $\begin{array}{llll}10403 & 20723 & 10878 & 44\end{array}$ & 06 & $\begin{array}{lll}11003 \quad 68161 & 90407 & 28\end{array}$ & 02 \\
\hline 8 & $\begin{array}{lllll}65536 & 16938 & 67090 & 17\end{array}$ & 05 & $\begin{array}{llll}10000 & 42589 & 27890 & 58\end{array}$ & 02 \\
\hline $23 / 3$ & $412852758234437 \quad 32$ & 05 & $\begin{array}{l}90895 \quad 31887 \quad 0430492 \\
\end{array}$ & 01 \\
\hline $22 / 3$ & $\begin{array}{lllll}26008 & 17634 & 03920 & 72\end{array}$ & 05 & $\begin{array}{llll}82626 & 93498 & 64416 & 39\end{array}$ & 01 \\
\hline 7 & $1638421345 \quad 5199572$ & 05 & $\begin{array}{llll}75123 & 97920 & 96579 & 80\end{array}$ & 01 \\
\hline $20 / 3$ & $\begin{array}{llll}10321 & 50411 & 38500 & 09\end{array}$ & 05 & $\begin{array}{llll}68318 & 62538 & 56294 & 03\end{array}$ & 01 \\
\hline $19 / 3$ & $\begin{array}{lll}65022 & 44664 & 70976 \quad 70\end{array}$ & 04 & $\begin{array}{llll}62149 & 80828 & 62956 & 03\end{array}$ & 01 \\
\hline 6 & $\begin{array}{l}4096270948 \quad 06401 \quad 08\end{array}$ & 04 & $\begin{array}{lllll}56562 & 77857 & 28816 & 43\end{array}$ & 01 \\
\hline $17 / 3$ & $\begin{array}{llll}25806 & 12469 & 70992 & 37\end{array}$ & 04 & $\begin{array}{llll}51508 & 76680 & 73079 & 38\end{array}$ & 01 \\
\hline $16 / 3$ & $\begin{array}{llll}16258 & 18668 & 37913 & 66\end{array}$ & 04 & $\begin{array}{llll}46944 & 78141 & 49920 & 25\end{array}$ & 01 \\
\hline 5 & 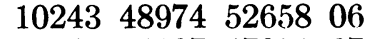 & 04 & $\begin{array}{llll}42833 & 58575 & 64239 & 47\end{array}$ & 01 \\
\hline $14 / 3$ & $\begin{array}{lllll}64546 & 14107 & 47051 & 67\end{array}$ & 03 & $\begin{array}{llll}39143 & 93590 & 59400 & 43\end{array}$ & 01 \\
\hline $13 / 3$ & $\begin{array}{llll}40679 & 42509 & 60218 & 28\end{array}$ & 03 & 35851229603511528 & 01 \\
\hline
\end{tabular}


TABLE 1-Continued

\begin{tabular}{|c|c|c|c|c|}
\hline$s$ & $\zeta\left(s, \frac{1}{4}\right)$ & & $\zeta\left(s, \frac{3}{4}\right)$ & \\
\hline 4 & $\begin{array}{llll}25646 & 36906 & 68198 & 07\end{array}$ & 03 & $32938 \quad 854224751000$ & 01 \\
\hline $11 / 3$ & $\begin{array}{llll}16178 & 62720 & 13961 & 71\end{array}$ & 03 & $\begin{array}{lll}30400 & 81320 & 64265 \quad 08\end{array}$ & 01 \\
\hline $10 / 3$ & $102174813983634 \quad 30$ & 03 & $\begin{array}{llll}28246 & 87653 & 98801 & 74\end{array}$ & 01 \\
\hline 3 & $646638699687684 \quad 60$ & 02 & $\begin{array}{lllll}26513 & 16608 & 16881 & 98\end{array}$ & 01 \\
\hline $8 / 3$ & 41092641687544799 & 02 & $\begin{array}{lllll}25285 & 74853 & 60394 & 04\end{array}$ & 01 \\
\hline $7 / 3$ & $26334 \quad 77863 \quad 02380 \quad 64$ & 02 & $\begin{array}{llll}24759 & 9605502275 & 82\end{array}$ & 01 \\
\hline 2 & $\begin{array}{llll}17197 & 32915 & 45071 & 11\end{array}$ & 02 & $\begin{array}{llll}25418 & 79647 & 67160 & 65\end{array}$ & 01 \\
\hline $5 / 3$ & $11787 \quad 30284 \quad 47967 \quad 72$ & 02 & $\begin{array}{llll}28747 & 02273 & 10830 & 06\end{array}$ & 01 \\
\hline $\begin{array}{l}4 / 3 \\
1\end{array}$ & $\begin{array}{c}9567633344 \underset{\infty}{56813} 25 \\
\end{array}$ & 01 & $\begin{array}{c}4223101605 \underset{\infty}{70504} 20 \\
.\end{array}$ & 01 \\
\hline $2 / 3$ & -24411861449688978 & 00 & -20381061318246556 & 01 \\
\hline $1 / 3$ & 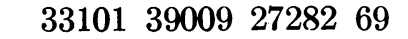 & 00 & $\begin{array}{llll}-64976 & 99170 & 75290 & 43\end{array}$ & 00 \\
\hline 0 & 25000000000000000 & 00 & -25000000000000000 & 00 \\
\hline$-1 / 3$ & $1349919957 \quad 7966553$ & 00 & -89579844838811398 & -01 \\
\hline$-2 / 3$ & $\begin{array}{llll}55399 & 96817 & 77458 & 36\end{array}$ & -01 & $-19221 \quad 97815 \quad 5428852$ & -01 \\
\hline-1 & $10416 \quad 66666 \quad 66666 \quad 67$ & -01 & $\begin{array}{llll}10416 & 66666 & 66666 & 67\end{array}$ & -01 \\
\hline$-4 / 3$ & $-1055877278 \quad 3612400$ & -01 & $2014785800 \quad 0231975$ & -01 \\
\hline$-5 / 3$ & $-1699251822 \quad 2981252$ & -01 & 20093864255483596 & -01 \\
\hline-2 & -15625000000000000 & -01 & 15625000000000000 & -01 \\
\hline$-7 / 3$ & $-10834 \quad 450589550842$ & -01 & $\begin{array}{lllll}97268 & 16010 & 89067 & 10\end{array}$ & -02 \\
\hline$-8 / 3$ & -52815718787000444 & -02 & $\begin{array}{lllll}40704 & 00253 & 32719 & 07\end{array}$ & -02 \\
\hline-3 & $-4557291666 \quad 66666 \quad 67$ & -03 & $\begin{array}{llll}-45572 & 91666 & 66666 & 67\end{array}$ & -03 \\
\hline$-10 / 3$ & $29301 \quad 123948050984$ & -02 & $\begin{array}{llll}-34597 & 28641 & 05474 & 40\end{array}$ & -02 \\
\hline$-11 / 3$ & $\begin{array}{lllll}46698 & 19500 & 95810 & 35\end{array}$ & -02 & $-48805 \quad 589038831547$ & -02 \\
\hline-4 & $48828 \quad 12500 \quad 00000 \quad 00$ & -02 & $-48828 \quad 12500 \quad 0000000$ & -02 \\
\hline$-13 / 3$ & $\begin{array}{lllll}38899 & 36684 & 63823 & 16\end{array}$ & -02 & $\begin{array}{llll}-37822 & 44498 & 22381 & 09\end{array}$ & -02 \\
\hline$-14 / 3$ & $2123995040 \quad 97140 \quad 41$ & -02 & $-19861 \quad 15110 \quad 11172 \quad 00$ & -02 \\
\hline-5 & $60066 \quad 344246031746$ & -04 & $\begin{array}{llll}60066 & 34424 & 60317 & 46\end{array}$ & -04 \\
\hline$-16 / 3$ & -18432750298301406 & -02 & $\begin{array}{llll}19233 & 18930 & 79122 & 45\end{array}$ & -02 \\
\hline$-17 / 3$ & -31960868109837130 & -02 & $\begin{array}{llll}32323 & 03687 & 52690 & 05\end{array}$ & -02 \\
\hline-6 & -37231445312500000 & -02 & $372314453124500 \quad 00$ & -02 \\
\hline$-19 / 3$ & $-33017 \quad 117057024087$ & -02 & $\begin{array}{llll}32782 & 87245 & 91040 & 61\end{array}$ & -02 \\
\hline$-20 / 3$ & -19865271459791151 & -02 & 19530955929821326 & -02 \\
\hline-7 & $-16148 \quad 88509 \quad 11458 \quad 33$ & -04 & $-16148 \quad 88509 \quad 11458 \quad 33$ & -04 \\
\hline$-22 / 3$ & $22055 \quad 3781757697 \quad 42$ & -02 & $-2229283934 \quad 1678100$ & -02 \\
\hline$-23 / 3$ & $4157748345 \quad 17227 \quad 95$ & -02 & $-41695 \quad 517684832096$ & -02 \\
\hline-8 & $52833 \quad 5571289062 \quad 50$ & -02 & $-52833 \quad 557128906250$ & -02 \\
\hline$-25 / 3$ & $50987 \quad 50282 \quad 80912 \quad 53$ & -02 & 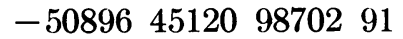 & -02 \\
\hline$-26 / 3$ & $3322998874 \quad 70615 \quad 11$ & -02 & -33088823879466838 & -02 \\
\hline-9 & $73837 \quad 51146 \quad 72111 \quad 74$ & -05 & $7383751146 \quad 7211174$ & -05 \\
\hline$-28 / 3$ & 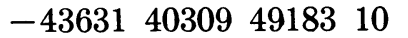 & -02 & $\begin{array}{llll}43748 & 62335 & 98758 & 29\end{array}$ & -02 \\
\hline$-29 / 3$ & $179840 \quad 4945597$ & -6 & $88394 \quad 53105 \quad 86276 \quad 23$ & -02 \\
\hline-10 & $-12045 \quad 14503 \quad 4790039$ & 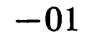 & $\begin{array}{lllll}12045 & 14503 & 47900 & 39\end{array}$ & -01 \\
\hline$-31 / 3$ & -12448123264593315 & -01 & $\begin{array}{llll}12442 & 55605 & 16962 & 73\end{array}$ & -01 \\
\hline$-32 / 3$ & -86654891907047886 & -02 & $\begin{array}{llll}86562 & 63379 & 78600 & 91\end{array}$ & -02 \\
\hline & -51470939638559344 & - & -51470939638559344 & -05 \\
\hline$-34 / 3$ & -12956249179849548 & -0 & - 12964947378990323 & -01 \\
\hline$-35 / 3$ & $27851 \quad 69352 \quad 45565 \quad 33$ & -01 & -27856 639442421263 & -01 \\
\hline-1 & $40274 \quad 33693 \quad 40896 \quad 61$ & -01 & -40274336934089661 & -01 \\
\hline & $\begin{array}{llll}44066 & 74225 & 89134 & 15\end{array}$ & - & $-44061 \quad 8130066818 \quad 43$ & -01 \\
\hline & 32424262873838585 & -( & $-32415 \quad 62726 \quad 12390 \quad 43$ & -01 \\
\hline-1 & $\begin{array}{llll}50856 & 42139 & 11692 & 30\end{array}$ & -8 & $\begin{array}{lllll}50856 & 42139 & 11692 & 30\end{array}$ & -05 \\
\hline$-40 / 3$ & -53981951603560958 & -0 & $\begin{array}{lllll}53991 & 01083 & 71719 & 25\end{array}$ & 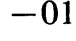 \\
\hline
\end{tabular}


TABLE 1-Continued

\begin{tabular}{|c|c|c|c|c|}
\hline$s$ & \multicolumn{2}{|l|}{$\zeta\left(s, \frac{1}{4}\right)$} & \multicolumn{2}{|l|}{$\zeta\left(s, \frac{3}{4}\right)$} \\
\hline$-4 i / 3$ & $-12213 \quad 66720 \quad 37044 \quad 43$ & 00 & $12214 \quad 20945 \quad 6595194$ & 00 \\
\hline-14 & -18566938210278749 & 00 & $\begin{array}{llll}18566 & 93821 & 02787 & 49\end{array}$ & 00 \\
\hline$-43 / 3$ & -21331387082289245 & 00 & $2133079048 \quad 86111 \quad 17$ & 00 \\
\hline$-44 / 3$ & $\begin{array}{llll}-16461 & 18663 & 02940 & 22\end{array}$ & 00 & $\begin{array}{llll}16460 & 09042 & 37713 & 87\end{array}$ & 00 \\
\hline-15 & -67634014389934201 & -05 & -67634014389934201 & -05 \\
\hline$-46 / 3$ & $\begin{array}{lll}30053 & 84266 \quad 68572 \quad 82\end{array}$ & 00 & $\begin{array}{llll}-30055 & 10353 & 95330 & 80\end{array}$ & 00 \\
\hline$-47 / 3$ & $\begin{array}{llll}71088 & 87471 & 07870 & 00\end{array}$ & 00 & -71089663757397933 & 00 \\
\hline-16 & $11287 \quad 34563 \quad 53554 \quad 50$ & 01 & -11287345635355450 & 01 \\
\hline$-49 / 3$ & $\begin{array}{llll}13532 & 13201 & 24995 & 85\end{array}$ & 01 & $-13532 \quad 03739 \quad 35347 \quad 35$ & 01 \\
\hline$-50 / 3$ & 10887193012397026 & 01 & $-1088701175 \quad 1445329$ & 01 \\
\hline-17 & $\begin{array}{llll}11649 & 82235 & 12560 & 03\end{array}$ & -04 & $\begin{array}{lll}11649 & 82235 \quad 12560 \quad 03\end{array}$ & -04 \\
\hline$-52 / 3$ & $\begin{array}{llll}-21552 & 81617 & 38443 & 47\end{array}$ & 01 & $\begin{array}{llll}21553 & 04222 & 79286 & 09\end{array}$ & 01 \\
\hline$-53 / 3$ & $-5302089805 \quad 5258377$ & 01 & $\begin{array}{llll}53021 & 04518 & 12564 & 35\end{array}$ & 01 \\
\hline-18 & -87489012928599550 & 01 & $874890129285995 \quad 50$ & 01 \\
\hline$-55 / 3$ & -10892532795344203 & 02 & $10892 \quad 51375 \quad 45982 \quad 56$ & 02 \\
\hline$-56 / 3$ & 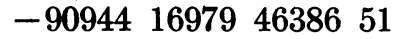 & 01 & $90943 \quad 7912583854 \quad 77$ & 01 \\
\hline-19 & $-2523056188 \quad 5826209$ & -04 & $-25230 \quad 561885826209$ & -04 \\
\hline$-58 / 3$ & 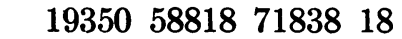 & 02 & -19350638926170453 & 02 \\
\hline$-59 / 3$ & $4930435983 \quad 5405891$ & 02 & -49304394038705558 & 02 \\
\hline-2 & $\begin{array}{llll}84212 & 65834 & 78432 & 17\end{array}$ & 02 & $\begin{array}{llll}-84212 & 65834 & 78432 & 17\end{array}$ & 02 \\
\hline$-61 / 3$ & $\begin{array}{lllll}10846 & 36397 & 60161 & 63\end{array}$ & 03 & -10846359235997600 & 03 \\
\hline
\end{tabular}


TABLE 2

\begin{tabular}{|c|c|c|c|c|c|c|c|c|}
\hline$s$ & \multicolumn{3}{|c|}{$\zeta(s)$} & $s$ & \multicolumn{4}{|c|}{$\zeta(s)$} \\
\hline $64 / 3$ & 1000000378 & 5323325 & 01 & $31 / 3$ & 10007 & 87512 & 5973522 & 01 \\
\hline 21 & 1000000476 & $93298 \cdot 68$ & 01 & 10 & 10009 & 94575 & 1278181 & 01 \\
\hline $62 / 3$ & 1000000600 & 9154153 & 01 & $29 / 3$ & 10012 & 56544 & 8949633 & 01 \\
\hline $61 / 3$ & 1000000757 & 1312399 & 01 & $28 / 3$ & 10015 & 88198 & 6230652 & 01 \\
\hline 20 & 1000000953 & $96203 \quad 39$ & 01 & & 10020 & 08392 & 8260822 & 01 \\
\hline $59 / 3$ & 1000001201 & $96944 \quad 13$ & 01 & $26 / 3$ & 10025 & 41240 & $15797 \quad 39$ & 01 \\
\hline $58 / 3$ & 1000001514 & 4624987 & 01 & $25 / 3$ & 10032 & 17649 & $68406 \quad 42$ & 01 \\
\hline 19 & 1000001908 & 2127166 & 01 & & 10040 & 77356 & $19794 \quad 43$ & 01 \\
\hline $56 / 3$ & 1000002404 & 3554591 & 01 & $23 / 3$ & 10051 & 71613 & 2389226 & 01 \\
\hline $55 / 3$ & 1000003029 & 5262390 & 01 & $22 / 3$ & 10065 & 66797 & 0811032 & 01 \\
\hline 18 & $10000 \quad 03817$ & 2932650 & 01 & & 10083 & 49277 & 3819228 & 01 \\
\hline $53 / 3$ & 1000004809 & 9636494 & 01 & $20 / 3$ & 10106 & 32075 & 3204838 & 01 \\
\hline $52 / 3$ & 1000006060 & 8609824 & 01 & $19 / 3$ & 10135 & 64087 & 2187002 & 01 \\
\hline 17 & $10000 \quad 07637$ & 1976379 & 01 & 6 & 10173 & 43061 & 9844491 & 01 \\
\hline $50 / 3$ & 1000009623 & $69761 \quad 94$ & 01 & $17 / 3$ & 10222 & 34193 & 4594971 & 01 \\
\hline $49 / 3$ & 1000012127 & 1668042 & 01 & $16 / 3$ & 10285 & 97325 & 5286825 & 01 \\
\hline 16 & 1000015282 & 2594087 & 01 & 5 & 10369 & 27755 & 1433699 & 01 \\
\hline $47 / 3$ & 1000019258 & 7553322 & 01 & $14 / 3$ & 10479 & 19229 & $28351 \quad 53$ & 01 \\
\hline $46 / 3$ & 1000024270 & $74650 \quad 59$ & 01 & $13 / 3$ & 10625 & 64589 & $86109 \quad 19$ & 01 \\
\hline 15 & 1000030588 & 2363070 & 01 & 4 & 10823 & 23233 & 7111382 & 01 \\
\hline $44 / 3$ & 1000038551 & $79067 \quad 62$ & 01 & $11 / 3$ & 11094 & 13692 & $59748 \quad 37$ & 01 \\
\hline $43 / 3$ & 1000048591 & $\begin{array}{lll}05077 & 17\end{array}$ & 01 & $10 / 3$ & 11473 & 56236 & 8827429 & 01 \\
\hline 14 & 1000061248 & 1350587 & 01 & 3 & 12020 & 56903 & 1595943 & 01 \\
\hline $41 / 3$ & 1000077207 & $23697 \quad 67$ & 01 & $8 / 3$ & 12841 & 90540 & $23974 \quad 21$ & 01 \\
\hline $40 / 3$ & 1000097332 & 0800025 & 01 & $7 / 3$ & 14151 & 55609 & 4459830 & 01 \\
\hline 13 & 1000122713 & 3475785 & 01 & 2 & 16449 & 34066 & 8482264 & 01 \\
\hline $38 / 3$ & 1000154728 & $79053 \quad 14$ & 01 & $5 / 3$ & 21235 & 22968 & 8575835 & 01 \\
\hline $37 / 3$ & 1000195119 & 4682160 & 01 & $4 / 3$ & 36009 & 37750 & 4588624 & 01 \\
\hline 12 & 1000246086 & 5533080 & 01 & 1 & & $\infty$ & & \\
\hline $35 / 3$ & 1000310414 & 3928700 & 01 & $2 / 3$ & -24475 & 80736 & 2336582 & 01 \\
\hline $34 / 3$ & 1000391627 & 1627387 & 01 & $1 / 3$ & -97336 & 02483 & 5078272 & 01 \\
\hline 11 & 1000494188 & 6041195 & 01 & 0 & -50000 & 00000 & 0000000 & 00 \\
\hline $32 / 3$ & 1000623757 & 1625128 & 01 & & & & & \\
\hline
\end{tabular}

